\title{
Práticas experimentais de Física a distância: Desenvolvimento de uma aplicação com Arduino para a realização do Experimento de Millikan remotamente
}

\author{
Physics experimental practices in the distance: development of an application with arduino for the
} realization of the Millikan experiment remotely

\author{
Tiago R. Barros ${ }^{1}$, Wandearley S. Dias*10 \\ ${ }^{1}$ Universidade Federal de Alagoas, Instituto de Física, Maceió, AL, Brasil
}

Recebido em 28 de Fevereiro, 2019. Aceito em 03 de Maio, 2019.

\begin{abstract}
Apresentamos a elaboração de um sistema que permite o acesso remoto e controle didático de um experimento de física através da internet. Mais especificamente, descrevemos um sistema de instrumentação remota para determinação do valor da carga elementar através do método de Millikan. O sistema é constituído de elementos físicos (hardware) e lógicos (software) integrados à uma plataforma Arduino para o controle e interfaceamento com o computador. A avaliação do protótipo foi realizada em dois testes, envolvendo um professor da rede pública de ensino médio e 14 alunos também da rede pública de ensino médio. Os resultados quantitativos e qualitativos apontam para a viabilidade de implementação do recurso didático.
\end{abstract}

Palavras-chave: Ensino a distância, instrumentação virtual, arduino, experimento remoto.

We present the construction of a system that allows remote access and didatic control of a physics experiment through the internet. More specifically, we describe a remote instrumentation system to determine of the value of the elementary charge by using the Millikan method. The system is composed of hardware and software integrated to an Arduino platform in order to control and communication with the computer. The evaluation of the prototype was conducted in two tests, involving a teacher from the public high school and 14 students also from the public high school. The quantitative and qualitative results point out to the feasibility of implementing the didatic resource.

Keywords: Teaching at a distance, virtual instrumentation, arduino, remote experiment.

\section{Introdução}

De maneira geral, o ensino de Física ainda é caracterizado pela abordagem baseada na resolução de vários exercícios repetidamente, muitas vezes resolvidos mecanicamente, fazendo uso de uma sucessão de equações. Estas "fórmulas" são muitas vezes memorizadas, desprezando assim uma análise mais profunda, onde a compreensão dos fenômenos físicos envolvidos seria o objetivo principal. Assim, o modelo de ensino fudamentado na transmissão do conhecimento, onde o aluno atua como agente passivo, tem se mostrado pouco efetivo para uma aprendizagem significativa da ciência.

Insatisfações com o ensino de física não são recentes nem restritas ao nosso país. Em meados do século passado, propostas como o curso de física do Physical Science Study Committee (Estados Unidos), o Nuffield Project (Inglaterra), o Harvard Physics Project (Estados Unidos), o projeto de Ensino de Física (Brasil) e Física Auto-Instrutiva (Brasil), surgiram com a proposta de abordar os conteúdos físicos de uma maneira menos abs-

*Endereço de correspondência: wandearley@fis.ufal.br trata que os livros da época, acompanhados da ênfase na componente prático-experimental $\sqrt{1-6}$. No que se refere ao contexto brasileiro, embora elaborados e interessantes, os projetos mostraram-se inviáveis à realidade educacional daquela época. Relata-se que, após alguns anos, pouca ou nenhuma difusão foi constatada na rede escolar [3, 4]. Aponta-se como motivo a falta de concepção de aprendizagem, uma vez que explorou-se muito o "como ensinar física" e pouco ou nada sobre "como aprender física" 3 .

Vale ressaltar que, embora os resultados alcançados pelos referidos projetos não tenham sido aqueles desejados, tais projetos podem ter sido precursores da pesquisa em ensino de física. Aparecendo com mais clareza nos anos setenta, tais pesquisas tem explorado, desde então, diferentes seguimentos como concepções alternativas 7], mudança conceitual [8], representações mentais dos alunos [9], equipamentos de baixo custo 10 e história e filosofia da ciência 11.

Apesar dos esforços, uma mudança no cenário atual ainda é um dos grandes desafios no processo de ensino e aprendizagem de física, motivando diferentes estudos 
e práticas docentes 12 17]. Entre as propostas, vários autores destacam a importância da abordagem práticoexperimental no processo de ensino-aprendizagem de física, ganhando muitas vezes conotação de imprescindibilidade [18]. Isso não significa evidenciar a primazia da experimentação na construção do conhecimento, e sim reconhecer que estas atividades podem contribuir significativamente para a melhoria da compreensão de conceitos físicos, assim como desenvolver o raciocínio lógico e a comunicação, estimular a capacidade de iniciativa e de trabalho em grupo, motivar e desenvolver uma aprendizagem ativa 19 21. Mais importante que a memorização de equações, conceitos e leis que regem a física, é a construção de argumentos que justificam os resultados observados. Nesse sentido teremos um modelo de ensino mais congruente com a gênese da ciência e do fazer científico 22 .

O uso de experimentos como ferramenta educacional apresenta concordância com algumas teorias de aprendizagem. Sob a luz de Piaget, o uso de experimentos é uma oportunidade de rever e contrapor a teoria estudada em sala ou abstrair novas informações que a experiência propicia 23 24. É um momento de desequilíbrio, onde há o confronto entre as estruturas congnitivas e o que é real, promovendo então um reajuste conceitual ou construção de um novo esquema mental. Por outro lado, sob o olhar de Vigotski, o professor pode atuar como parceiro experiente, promovendo o estímulo de interações sociais dentro da zona de desenvolvimento proximal do aprendiz 25 26]. Desta forma, além de facilitar o processo de interação social entre professor e aluno, o uso de experimentos possibilita que essa interação seja mais significativa para a aprendizagem. Dentro da teoria cognitivista de Ausubel, os experimentos podem atuar como organizadores prévios, elementos motivadores usados para facilitar a aprendizagem subsequente 27,28 . Neste caso, a disposição para aprender, pois influencia a percepção do aluno em relação ao objeto de estudo.

Contudo, existem dificuldades para a implementação de aulas com uso de experimentos ou prático-experimentais. Infraestrutura ausente ou inadequada [29], ausência de materiais ou equipamentos [30], baixa remuneração dos professores e tempo disponível para preparação de aulas 31, bem como o despreparo do docente para planejar e dirigir este tipo de atividade [32], apresentam-se como aspectos reportados. Essas dificuldades podem ser amplificadas se analisadas no contexto de Educação a Distância $(\mathrm{EaD})$, onde há relatos de aulas com caráter presencial, concentradas em finais de semana 33. A necessidade de constantes deslocamentos dos estudantes em direção aos pólos para a realização das atividades experimentais diminui a possibilidade de se alcançar aqueles que se encontram mais marginalizados e excluídos do sistema formal de ensino 34. Em seu trabalho, Veloso et. al. 35 relata os formatos de laboratórios didáticos utilizados na EaD, onde encontram-se cursos apoiados em materiais impressos, kits de experimentos com guias para montar e testar, bem como aqueles que contam com a utilização de simuladores. De fato, em meio as dificuldades apresentadas, animações e simulações computacionais de experimentos de física têm sido bastante exploradas 33, 36 39]. Entretanto, é preciso reconhecer estas atividades como mediações distintas, sendo mais uma estratégia didática, que pode ser complementar ao experimento físico [36]. Assim, é importante que professores e alunos estejam conscientes de que eles são um modelo simplificado da realidade, sob risco de assimilar uma ideia errada do fenômeno em estudo [39.

Dentro deste contexto, destacamos o interesse recente no desenvolvimento de protótipos que possibilitam a experimentação à distância $21,40,44]$. Experimentos controlados remotamente se mostram uma boa ferramenta de ensino, facilitando o processo de interação social entre aluno e professor, bem como se tornando alternativa aos professores que não dispõem de infraestrutura necessária nas escolas em que atuam para realizarem atividades de caráter investigativo em seus cursos [41]. Entedemos que controlar remotamente os experimentos não deve ser limitado ao olhar didático, mas também como interessante ferramenta interdiciplinar, capaz de trazer proximidade entre as ciências e promover integração tecnológica.

Acreditando no potencial desta metodologia para melhoria do processo de ensino/aprendizagem de física, nós apresentamos o desenvolvimento, a baixo custo, de um sistema de controle que possibilita a gerência experimental remotamente. Mais especificamente, tratamos no presente artigo a elaboração e realização experimental remota para determinação da carga elementar pelo método de Millikan. A escolha do tema está relacionada à sua relevância junto ao desenvolvimento científico do início do século passado, sendo um dos clássicos experimentos frequentemente presentes em laboratórios de física moderna, bem como em abordagens sobre a História e Filosofia da Ciência 45. Entendemos que a viabilidade da nossa proposta estende o acesso à experimentação, possibilitando a abrangência na formação científica das novas gerações.

O presente artigo está dividido da seguinte forma: $\mathrm{Na}$ seção 2 apresentamos os conceitos físicos presentes no experimento. Na seção 3 descrevemos a estrutura do experimento, tratando a composição de hardware e software do mesmo. Submetemos o protótipo experimental a dois testes: Um professor e 14 alunos da rede pública de ensino médio do estado. Apresentamos na seção 4 resultados quantitativos e qualitativos sobre a implementação do protótipo. Por fim, na seção 5 apresentaremos nossas considerações finais.

\section{Sobre o experimento}

A Figura 1 apresenta uma versão simplificada do experimento. Gotas de óleo carregadas eletricamente são inseridas entre um par de placas paralelas horizontais. Através de uma fonte, um campo elétrico uniforme é esta- 




Figura 1: Diagrama esquemático do experimento de Millikan. Após a pulverização, gotas de óleo eletricamente carregadas penetram numa região entre placas paralelas horizontais ligadas a uma fonte que estabelece uma diferença de potencial elétrica $(V)$ entre elas. O sentido da força elétrica que atua sobre a gotícula é controlada pela polaridade das placas, enquanto o movimento das gotículas pode ser acompanhado pela luneta.

belecido entre este par de placas paralelas, fazendo com que uma força elétrica atue sobre as gotículas. Ao alterar a polaridade das placas, altera-se também o sentido da força elétrica atuando sobre as gotículas. Dessa forma, as gotículas podem apresentar um movimento de subida ou descida, sendo este processo controlado pelo usuário. Tendo em mente a utilização de uma escala graduada acoplada à luneta de observação, a medida do tempo que uma determinada gotícula necessita para se deslocar entre duas marcas da escala graduada pode ser utilizada para estabelecer a velocidade desta gotícula.

A análise física revela quatro forças relevantes atuando sobre as gotículas: força elétrica $\left(\vec{F}_{E}\right)$, força peso $\left(\vec{F}_{g}\right)$, empuxo $(\vec{E})$ e a força viscosa $\left(\vec{F}_{v}\right)$. A força viscosa, que surge quando um corpo (gotícula) se movimenta no interior de um fluido, atua como uma força de atrito que tende a reduzir a velocidade do corpo. Esta força, que é proporcional à velocidade, é estabelecida através da lei de Stokes. Considerando um regime de baixas velocidades e que as gotículas são esféricas, podemos escrever força viscosa como:

$$
\left|\vec{F}_{v}\right|=6 \pi r \eta v
$$

onde $r$ é o raio da gotícula, $\eta$ é a viscosidade de fluido e $v$ é a velocidade da gotícula. A força peso é dada por

$$
\left|\vec{F}_{g}\right|=\rho_{1} V g
$$

onde $\rho_{1}$ é a densidade da gota, $V$ é o seu volume e $g$ é a aceleração da gravidade. O empuxo é estabelecido por

$$
|\vec{E}|=\rho_{2} V g
$$

onde $\rho_{2}$ é a densidade do fluido (Ar) e $V$ é o volume da gota. Por fim, a força elétrica

$$
\left|\vec{F}_{E}\right|=q \frac{\mathbb{V}}{d}
$$

onde $q$ é a carga da gotícula, $\mathbb{V}$ é diferença de potencial entre as placas paralelas e $d$ a distância entre elas.
Através do diagrama de forças para os movimentos descendente (Figura 2a) e ascendente (Figura 2b) é possível obter as equações:

$$
|\vec{E}|+\left|\vec{F}_{v}\right|-\left|\vec{F}_{E}\right|-\left|\vec{F}_{g}\right|=-\left|\vec{F}_{r}\right|_{\text {desc }}
$$

e

$$
|\vec{E}|+\left|\vec{F}_{E}\right|-\left|\vec{F}_{v}\right|-\left|\vec{F}_{g}\right|=\left|\vec{F}_{r}\right|_{a s c}
$$

Entre as partículas presentes na janela de visualização, recomenda-se escolher e acompanhar uma gotícula por vez. Em geral, indicamos que as gotículas com baixas velocidades apresentam-se, aproximadamente, em um movimento uniforme. Quando não apresentam, são facilmente identificadas. Este critério se faz importante, uma vez que estabelecemos que as gotículas selecionadas para análise devem apresentar um movimento uniforme na descida e subida. Neste cenário temos o regime de equilíbrio de forças, de maneira que força resultante $\left|\vec{F}_{r}\right|$ sobre a gotícula é nula. Assim, obtemos a velocidade da gotícula no movimento descendente

$$
v_{d}=\frac{1}{6 \pi r \eta}\left[q \frac{\mathbb{V}}{d}+\frac{4}{3} \pi r^{3} g\left(\rho_{1}-\rho_{2}\right)\right],
$$

assim como a velocidade ascendente

$$
v_{s}=\frac{1}{6 \pi r \eta}\left[q \frac{\mathbb{V}}{d}-\frac{4}{3} \pi r^{3} g\left(\rho_{1}-\rho_{2}\right)\right] .
$$

Como descrito anteriormente, estas velocidades são determinadas a partir da razão entre o espaço percorrido (medido através da escala graduada) e a respectiva medida de tempo. Desta forma, assumindo $g=9,81 \mathrm{~m} / \mathrm{s}^{2}$, os únicos parâmetros desconhecidos passam a ser a carga $q$ e o raio $r$ das gotículas.

A partir das equações 7 e 8 podemos encontrar uma expressão para a carga da gotícula em função do seu raio. Ainda manipulando tais equações, podemos também encontrar a expressão do raio da gotícula em função de

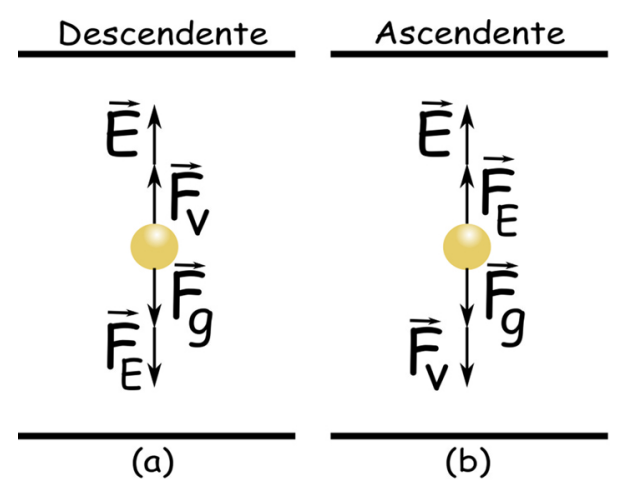

Figura 2: Representação gráfica das forças atuantes sobre uma gota de óleo no experimento de Millikan enquanto descreve um movimento (a) descentende e (b) ascendente. Aqui, $\vec{E}$ representa o empuxo, $\vec{F}_{v}$ a força viscosa, $\vec{F}_{E}$ a força elétrica e $\vec{F}_{g}$ a força peso. 
$\eta, g, v_{d}, v_{s}, \rho_{1}$ e $\rho_{2}$. A partir delas podemos encontrar

$$
q=\frac{9 \pi d\left(v_{d}+v_{s}\right)}{2 \mathbb{V}} \sqrt{\frac{\eta^{3}\left(v_{d}-v_{s}\right)}{g\left(\rho_{1}-\rho_{2}\right)}},
$$

onde temos a carga das gotículas dependendo apenas de parâmetros conhecidos ou obtidos experimentalmente.

É razoável pensar que, para descobrir o valor de $q$, poderíamos ajustar o valor de $\mathbb{V}$ (diferença de potencial entre as placas paralelas) até que a gotícula de óleo fique fixa. Assim, nossa análise de forças teriam apenas $\vec{F}_{g}, \vec{E}$ e $\vec{F}_{E}$. Entretanto, salientamos que este procedimento é muito difícil de se fazer na prática, justificando assim a metodologia anterior.

Para se estimar o valor da carga elétrica fundamental com precisão satisfatória estabelecemos a coleta de dados mínima de 15 gotículas. Cada gotícula selecionada (que atenda à condição supracitada) terá 5 intervalos de tempo registrados, tanto no movimento ascendente como também no movimento de descida. Os tempos são registrados em uma planilha, como exemplificado na Tabela 1. Salientamos que os resultados tendem a ficar melhores quanto maior for o número de amostras.

A ideia é verificar a existência de uma relação entre a carga $q$ e uma carga elementar $e$ conforme a expressão

$$
q=n e
$$

onde $n$ seria no número de cargas na gotícula. Um método consiste em tomar o menor valor de carga obtido como referência $\left(q_{r}\right)$ e dividir todos os valores de carga $q$ por $q_{r}$, obtendo assim uma quantidade que denotaremos de $n$. Organizando os dados em ordem crescente, podemos construir o gráfico $q$ versus $n$, onde a reta resultante traria como coeficiente angular o valor da carga elementar. Uma outra forma seria construir um histograma dos valores das cargas em função do número de ocorrências. Assim, é possível observar a existência de picos (maior ocorrência) como um múltiplo (inteiro) de uma quantidade bem definida. Seguindo o protocolo anterior, organizamos os dados em ordem crescente e encontramos o valor de $n$ correspondente a cada carga. Agora, podemos agrupar as partículas que apresentam valores de $n$ muito próximos e calcular o valor médio da carga elétrica $(\bar{q})$ de cada

Tabela 1: Planilha proposta para registro de valores dos tempos de subida e descida para cada gotícula.

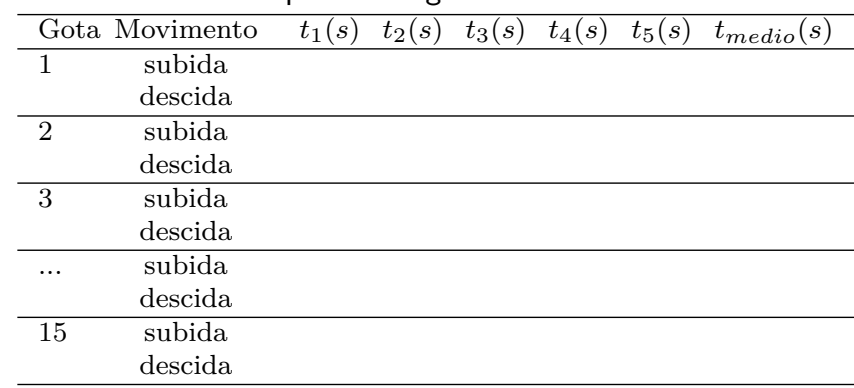

grupo $n$. Após a obtenção das diferenças entre dois valores consecutivos

$$
\Delta q=\bar{q}_{i+1}-\bar{q}_{i}
$$

a média aritmética das diferenças $\Delta q(\overline{\Delta q})$ indica um valor próximo do valor da carga fundamental encontrada na literatura.

\section{O aparato experimental}

O sistema proposto é formado pela integração de três sistemas: Aparato experimental (Hardware), dispositivo eletrônico que comanda a maquete experimental, e a interface que permite o controle remoto do dispositivo eletrônico (software).

\subsection{Hardware}

O experimento presente no laboratório de física moderna do Instituto de Física da UFAL já possibilitava a realização experimental presencialmente. Sua estrutura original, apresentada na Figura 3 , é similar à descrita anteriormente (Figura 1), sendo composta por uma pequena (a) câmara capacitiva, (b) um pulverizador de óleo, (c) uma luneta, (d) uma chave comutadora, (e) uma fonte elétrica e um cronômetro. Assim, em um experimento hands on, o usuário deverá produzir micro-gotas de óleo por meio do pulverizador. Estas gotas, que pelo atrito ou ionização do ar, adquirem carga elétrica, penetrarão na câmara capacitiva através do pequeno orifício na parte superior. Através da chave comutadora o usuário controla manualmente a inversão de polaridade nas placas da câmara capacitiva, observando através da luneta a resposta das micro-gotas à inversão do campo elétrico produzido pelas placas. Este último procedimento deve ser feito acompanhado de um um cronômetro, para aquisição dos tempos de subida e descida entre duas marcas.

Para que o experimento pudesse ser controlado remotamente, foi necessário a elaboração/implementação

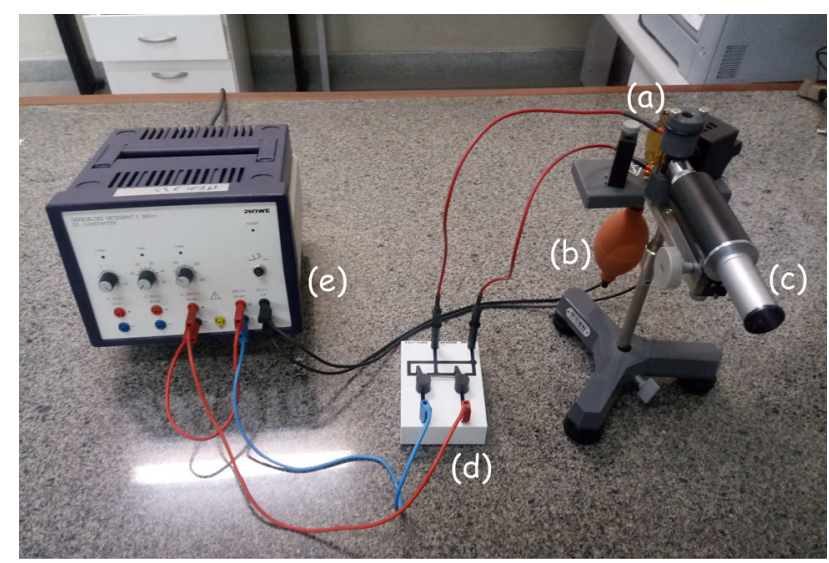

Figura 3: Aparato experimental disponível para realização do experimento de forma presencial. Na legenda, (a) indica a câmara capacitiva, (b) pulverizador de óleo, (c) luneta de observação, (d) chave comutadora, (e) fonte elétrica. 
de cinco dispositivos físicos adicionais: um computador, uma placa microcontroladora (plataforma Arduino); uma placa de circuito auxiliar, um compressor eletromecânico aliado a um tanque de armazenamento de ar; e uma câmera (tipo webcam). A câmera foi acoplada à luneta, permitindo o envio da imagem para o computador em tempo real. O compressor substitui o supracitado pulverizador. Por ser dotada de uma válvula eletromecânica, o controle remoto de geração das gotículas descritas anteriormente se torna possível. O diagrama elétrico do circuito de acionamento está representado na Figura 4 a. Este mecanismo conta ainda com um pressostato que faz o monitoramento da pressão no cilindro, acionando o compressor quando a pressão estiver abaixo da configurada. Desta forma, o cilindro sempre estará com pressão adequada para produção de gotículas. A placa de circuito auxiliar é composta por relés e transistores, com o objetivo de encaminhar os sinais enviados pelo microcontrolador para componentes do experimento. Neste circuito auxiliar contamos com o substituto elétrico da chave comutadora manual (ver diagrama elétrico na Figura 4 p). Todos os itens descritos estão associados à placa microcontroladora Arduino, permitindo a comunicação entre a maquete experimental e o computador/usuário. A plataforma Arduino é caracterizada por uma placa física associada a um microcontrolador e circuitos de

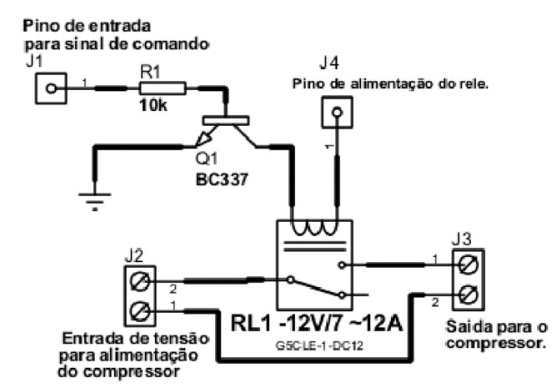

(a)

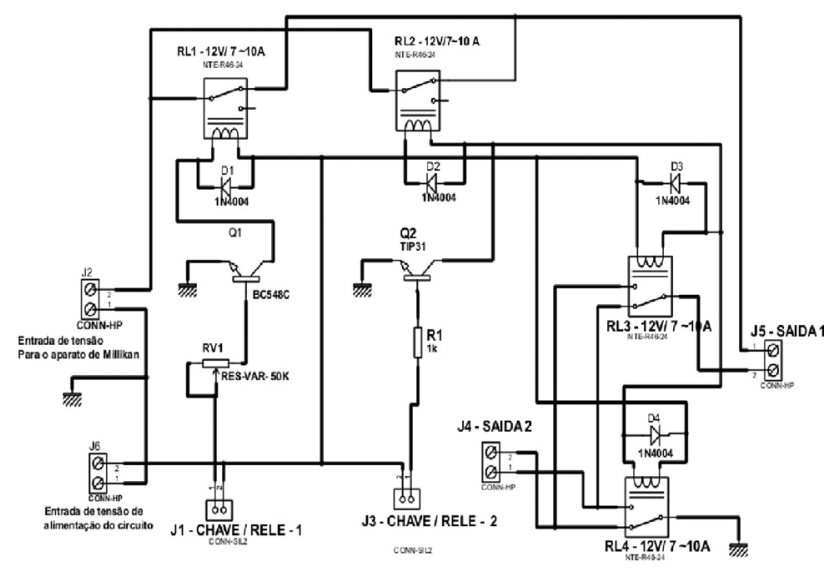

(b)

Figura 4: Diagrama elétrico (a) do circuito elétrico de acionamento do balão de ar para produção das gotículas e (b) do circuito de comutação da polaridade entre as placas do condensador. entrada e saída, cujas funcionalidades são desenvolvidas por meio da linguagem de programação padrão (baseada em $\mathrm{C} / \mathrm{C}++)$ [46, 47. Tais plataformas, conhecidas pelo seu baixo custo, programação relativamente fácil e enorme versatilidade, tem sido bastante utilizadas na automação de diversos projetos, inclusive para o ensino de física 41, 47,51. O computador, associado à placa Arduíno e à webcam, estabelece a conexão remota entre aparato experimental e usuário. Este computador atua como um servidor web, disponibilizando a interface gráfica para o usuário observar e controlar o experimento.

\subsection{Software}

O acesso remoto ao experimento supracitado via internet é estabelecido através do software de comunicação (TeamViewer), fazendo com que o usuário tenha acesso ao computador (servidor). Conectado ao servidor, o usuário tem acesso ao software de comando, um ambiente virtual desenvolvido para controle do protótipo experimental (ver Figura 5). Para o desenvolvimento do software de comando utilizamos a linguagem da programação Processing@, linguagem essa utilizada para o desenvolvimento de aplicações gráficas (imagens, animações, interações) e que possui uma interface de desenvolvimento integrada (IDE) bastante similar à do Arduíno [52,53].

Como pode ser visto na Figura 5 este ambiente conta com alguns itens: Através da representação gráfica do experimento (a), o aluno poderá visualizar o sentido das linhas de campo elétrico correspondente ao posicionamento da chave comutadora. Através dos botões "bolhas" (b) e "chave inversora" (c) ele poderá, respectivamente, produzir as gotículas e inverter a polaridade das placas condutoras. Através da janela de observação (d) e do cronômetro (e) ele poderá acompanhar o movimento das gotículas junto à escala graduada.

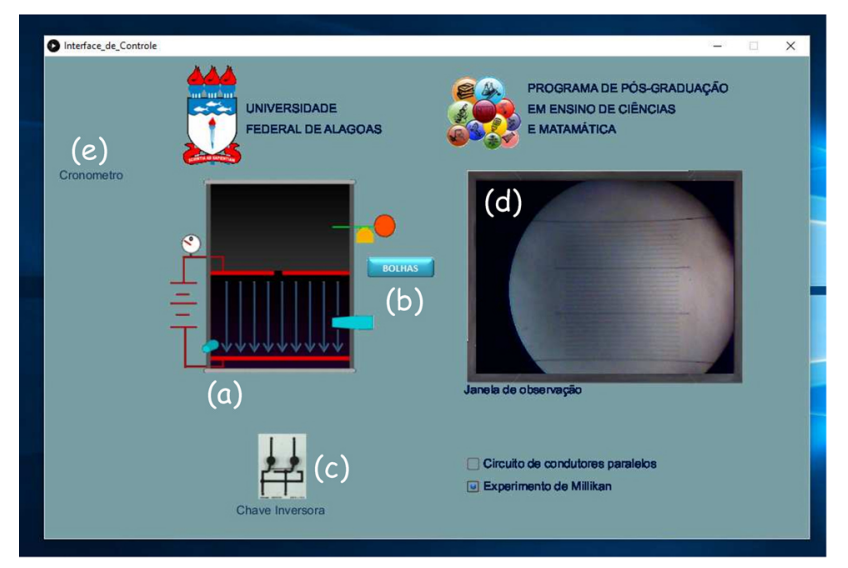

Figura 5: Interface gráfica do software de comando desenvolvido para realização do experimento remoto. $\mathrm{Na}$ legenda, ausente no modelo original, (a) indica a representação gráfica do experimento, (b) botão de bolhas, (c) botão da chave inversora, (d) janela de observação, (e) cronômetro em (ms). 
Na Figura 6 apresentamos uma representação gráfica da arquitetura lógica de comunicação entre maquete experimental e usuário. O usuário, munido de um computador com acesso à internet, conecta-se ao servidor por meio do software de comunicação. A partir daí o usuário terá acesso ao software de comando, que transmitirá ao aparato experimental os comandos requeridos pelo usuário através do circuito atuador. Paralelamente há o sistema de observação, permitindo a integração visual entre experimento e servidor/usuário.

\section{Experimentos testes}

Para verificação da proposta supracitada, submetemos o protótipo a dois testes, cada um com diferentes públicos alvo: um professor da rede pública do estado e alunos do terceiro ano do ensino médio da rede pública do estado (14 integrantes). O primeiro grupo caracteriza-se por professor do sexo masculino, 32 anos de idade, com formação acadêmica de licenciatura em física. O segundo grupo é composto por estudantes do sexo masculino e feminino, com idades entre 15 e 18 anos.

O protótipo experimental se manteve alocado no laboratório de física moderna do Instituto de Física da UFAL. Os computadores utilizados para acesso remoto do professor e estudantes estava alocado cerca de $30 \mathrm{~km}$ de distância. Antes da realização da prática experimental foram apresentadas a fundamentação teórica do experimento e as instruções para manuseio do equipamento. Para o professor, estes procedimentos demandaram aproximadamente 25 minutos. Em função da maturidade acadêmica dos integrantes do segundo grupo, foram necessários 120 minutos para estes procedimentos.

Para o desenvolvimento da prática experimental foi necessário dividir o segundo grupo em três equipes, visto que tínhamos apenas um protótipo para atendê-los. Para cada equipe foi disponibilizado um roteiro com procedimentos experimentais e orientações para análise. Todos tiveram como instrução obter, para cada gotícula, 5 medidas de tempo para os movimentos ascendente e descendente. Este procedimento foi realizado para 15 gotículas diferentes. Para a turma de alunos, cada equipe ficou resposável pela coleta de dados de 5 gotas.
O tempo de execução do experimento foi de aproximadamente 25 minutos, tanto para o professor como para os estudantes. Para o tratamento dos dados e apresentação dos resultados, o professor necessitou de aproximadamente 20 minutos, o segundo grupo cerca de 30 minutos (com auxílio do professor).

Após a análise dos dados, os resultados mostraram-se satisfatórios, com valores de $\overline{\Delta q} \approx 1,65 \times 10^{-19} C$ e $\overline{\Delta q} \approx 1,68 \times 10^{-19} C$. Para uma melhor compreensão dos dados coletados, apresentamos na Tabela 2 os dados de $q, \bar{q}, n$ e $\Delta q$ obtido pelos alunos. A organização desta planilha é pautada na descrição feita na seção 2, onde obtemos o valor de carga $q$ das gotículas a partir da equação 9 .

Além da análise dos dados, avaliamos alguns critérios qualitativos no tocante à proposta de experimentação remota. Observamos que um número maior de alunos exigiria mais tempo de acompanhamento do professor, ou seja, mais aulas. Uma possível solução seria ter à disposição mais de um protótipo experimental. Observamos ainda um grande interesse dos alunos, e também do professor, em realizar o procedimento experimental, apesar do manuseio ser indireto (via internet). As atitudes e

Tabela 2: Planilha com dados finais para determinação da carga elétrica fundamental a partir da coleta de dados dos alunos.

\begin{tabular}{cccc}
\hline$q(C)$ & $n$ & $\bar{q}(C)$ & $\Delta q(C)$ \\
\hline $1,42 \times 10^{-19}$ & 1,00 & & \\
$1,44 \times 10^{-19}$ & 1,01 & $1,44 \times 10^{-19}$ & \\
$1,46 \times 10^{-19}$ & 1,03 & & \\
\hline $2,07 \times 10^{-19}$ & 1,46 & $2,07 \times 10^{-19}$ & $6,31 \times 10^{-20}$ \\
\hline $2,22 \times 10^{-19}$ & 1,57 & & \\
& & $2,28 \times 10^{-19}$ & $2,10 \times 10^{-20}$ \\
$2,34 \times 10^{-19}$ & 1,65 & & \\
\hline $3,18 \times 10^{-19}$ & 2,24 & $3,18 \times 10^{-19}$ & $8,98 \times 10^{-20}$ \\
\hline $3,77 \times 10^{-19}$ & 2,66 & $3,77 \times 10^{-19}$ & $5,96 \times 10^{-20}$ \\
\hline $5,93 \times 10^{-19}$ & 4,18 & $5,93 \times 10^{-19}$ & $2,16 \times 10^{-19}$ \\
\hline $9,46 \times 10^{-19}$ & 6,66 & & \\
& & $9,69 \times 10^{-19}$ & $3,75 \times 10^{-19}$ \\
$9,91 \times 10^{-19}$ & 6,98 & & \\
\hline $1,12 \times 10^{-18}$ & 7,89 & $1,12 \times 10^{-18}$ & $1,52 \times 10^{-19}$ \\
\hline $1,35 \times 10^{-18}$ & 9,50 & $1,35 \times 10^{-18}$ & $2,29 \times 10^{-19}$ \\
\hline $1,47 \times 10^{-18}$ & 10,35 & $1,47 \times 10^{-18}$ & $1,21 \times 10^{-19}$ \\
\hline $1,82 \times 10^{-18}$ & 12,82 & $1,82 \times 10^{-19}$ & $3,51 \times 10^{-19}$ \\
\hline & & $\overline{\Delta q}(C)$ & $1,68 \times 10^{-19}$ \\
\hline
\end{tabular}

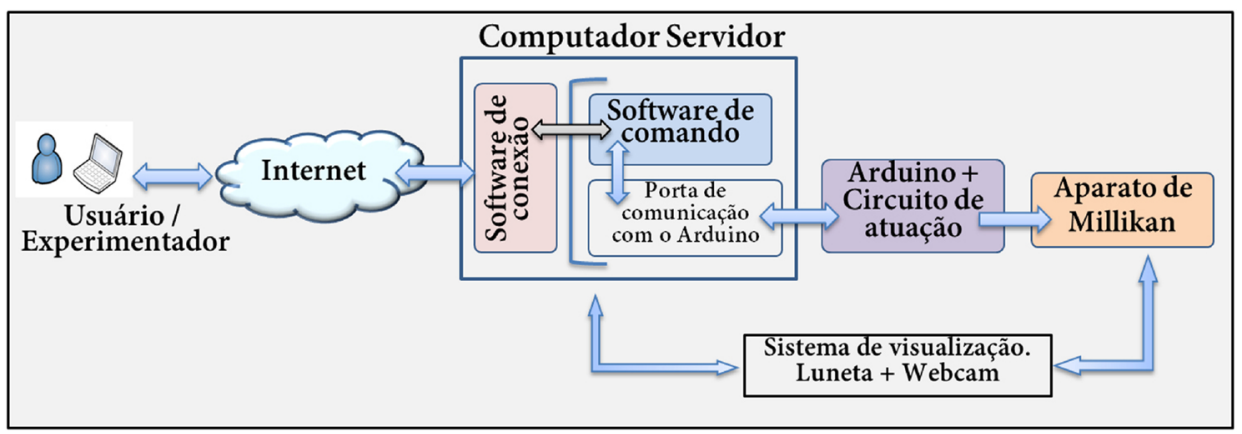

Figura 6: Representação gráfica da arquitetura lógica de comunicação entre usuário e protótipo experimental. 
comprometimento por parte do professor e dos estudantes nos leva a acreditar que a manipulação remota do experimento não deprecia a atividade experimental.

\section{Considerações finais}

Devemos ter ciência de que o ensino a distância para diferentes níveis foi autorizado por lei [54,55]. Apesar do seu crescimento, muitas disciplinas experimentais presentes nas grades curriculares dos cursos a distância ocorrem em aulas presenciais [33, limitando o alcance desta modalidade de ensino [34]. Assim, o desenvolvimento de tecnologias que permitam que estas aulas ocorram de forma não presencial se faz necessário. Diante dos aspectos legais e do baixo número de trabalhos acerca dessa categoria, acreditamos ser fundamental a ampliação de estudos acerca de atividades experimentais remotas. Dentro deste contexto, apresentamos a construção de um sistema que permite acessar e controlar remotamente um experimento de física para determinação da carga elementar através do método de Millikan. Para isso foram desenvolvidos elementos físicos (hardware) e lógicos (software), que integrados a uma plataforma Arduino, permitem o acesso e controle remoto do experimento através da internet. Após a construção, submetemos o protótipo a dois testes: com um professor da rede pública do ensino médio; com 14 alunos também da rede pública de ensino médio do estado. A análise técnica quantitativa nos mostrou resultados satisfatórios, onde os valores para carga elementar são bem próximos do valor conhecido na literatura. A análise qualitativa sugere que a manipulação remota do experimento não deprecia a atividade experimental. Diante dos resultados, consideramos que a ferramenta desenvolvida tem um alto grau de aplicabilidade.

\section{Agradecimentos}

Agradecemos à prof. Maria Teresa de Araújo, coordenadora dos laboratórios de ensino de física do IF/UFAL, pelo apoio e cooperação. Agradecemos às professoras Maria Socorro Seixas Pereira e Eleusa Ribeiro da Silva Dias pelas discussões e sugestões. Agradecemos à Gean da Silva Santos pelas sugestões e apoio técnico.

\section{Referências}

[1] U. Haber-Schaim, J.B. Cross, J.H. Dodge e J.A. Walter, PSSC Physics (D.C. Heath \& Co. Lexington, Massachusetts, 1960), $4^{\mathrm{a}}$ ed.

[2] M.A. Moreira e R. Axt, Rev. Ens. Fís. 8, 1 (1986).

[3] M.A. Moreira, Rev. Bras. Ens. Fis. 22, 1 (2000).

[4] F.L.A. Pena, Rev. Bras. Ens. Fis. 34, 1701 (2012).

[5] L.G. Barros, W. Cerqueira Júnior e G. Dutra, in $X$ Encontro Nacional de Pesquisa em Educação em Ciências (ABRAPEC, Águas de Lindóia, 2015).
[6] H.U. Gama e E.W. Hamburger, Pesquisas sobre o ensino de física (IFUSP, São Paulo, 1990).

[7] M.H. Gravina e B. Buchweitz, Rev. Bras. Ens. Fis. 16, 110 (1994).

[8] J.L.A. Pacca e A. Villani, Rev. Bras. Ens. Fis. 14, 222 (1992).

[9] A. Villani e L.O. Carvalho, Rev. Bras. Ens. Fis. 15, 74 (1993).

[10] R. Axt e M.A. Moreira, Rev. Bras. Ens. Fis. 13, 97 (1991).

[11] A. Morais e A. Guerra, Rev. Bras. Ens. Fis. 35, 1502 (2013).

[12] C.W.D. Rosa e A.B.D. Rosa, Revista Eletrônica de Ensenanza de las Ciências 4, 1 (2005).

[13] T.A. Borges, Caderno Brasileiro de Ensino de Física 19, 291 (2002).

[14] M. Fonseca, N.L. Maidana, E. Severino, S. Barros, G. Senhora e V.R. Vanin, Rev. Bras. Ens. Fis. 35, 1 (2013).

[15] V.B. Barbeta e I. Yamamoto, Rev. Bras. Ens. Fis. 24, $158(2002)$

[16] M.S.T. Araújo e M.L.V.D.S. Abibi, Rev. Bras. Ens. Fis. 25, 176 (2003).

[17] F. Catelli, Cad. Cat. Ens. Fís. 18, 108 (2001).

[18] J.P. Alves Filho, Cad. Cat. Ens. Fís. 17, 174 (2000).

[19] A. Hofstein e V.N. Lunetta, Science Education 88, 28 (2004).

[20] V.N. Lunetta, A. Hofstein e P. Clough, in: Handbook of Research in Science Education, editado por S.K. Abell e N.G.Lederman (Routledge, New Jersey, 2007).

[21] M.A.A. Monteiro, I.C.C. Monteiro, J.S.E. Germano e F. Sievers Junior, Cad. Bras. Ens. Fís. 30, 191 (2013).

[22] R. Driver, P. Newton e J. Osborne, Science Education 84, 3 (2000).

[23] J. Piaget, Psicologia e pedagogia (Rio de Janeiro, Forense, 1972).

[24] N. Piletti, Psicologia Educacional (Ática, São Paulo, 1999).

[25] L.S. Vigotsky, A formação social da mente: o desenvolvimento dos processos psicológicos superiores (Martins Fontes, São Paulo, 2007), $7^{a}$ ed.

[26] M.K. Oliveira, Vygotsky: aprendizado e desenvolvimento: um processo socio-histórico (Scipione, São Paulo, 1995), $3^{a}$ ed.

[27] D.P. Ausubel, J.D. Novak e H. Hanesian, Psicologia Educacional (Interamericana, Rio de Janeiro, 1980).

[28] C.R. Tironi, E. Schmit, V.R.N. Schumacher e E. Schumacher, in IX Encontro Nacional de Pesquisas em Educação em Ciências, (ABRAPEC, Aguas de Lindóia, 2013).

[29] T.R. Barros, Ensino de Física em Alagoas: Cenário Atual do Quadro de Professores de Física na Rede Estadual de Ensino, 2012. Trabalho de conclusão, Universidade Federal de Alagoas, Maceió, (2012).

[30] B.G. Kanbach, C.E. Laburu e O.H.M. Silva, in Anais do XIV Simpósio Nacional de Ensino de Física - SNEF (SBF, Rio de Janeiro, 2005).

[31] G.C. Lourencetti, Revista Educação Pública 23, 13 (2014).

[32] C.E. Laburú, M.A. Barros e B.G. Kanbach, Investigações em Ensino de Ciências 12, 305 (2007).

[33] N.C. Silva, Caderno Brasileiro de Ensino de Física 29, 1206 (2012). 
[34] V.B. Barbeta, in: Desafios da Educação a Distância na Formação de Professores (Secretaria de Educação a Distância, Brasilia, 2006), p. 150.

[35] M.S.S.O. Veloso e A.S. Andrade Neto, RENOTE - Revista Novas Tecnologias na Educação 12, 2 (2014).

[36] A. Medeiros, Rev. Bras. Ens. Fis. 24, 2 (2002).

[37] M.C. Linn, in: Mediating science learning through information and communications technology, editado por R. Holliman e E. Scanlon (RoutledgeFalmem, London, 2004).

[38] L. De La Torre, J. Sanches, S. Dormido, J.P. Sánchez, M. Yuste e M. Carreras, European Journal of Physics 32, 571 (2011).

[39] V. Heckler, M.F.O. Saraiva e K.S. Filho, Rev. Bras. Ens. Fis. 29, 267 (2007).

[40] M.C.R. Pessanha, S.G. Cozendey e M.O. Souza, Rev. Bras. Ens. Fis. 32, 4503 (2010).

[41] A.A. Sim, Experimento de Física Controlato Remotamente: uma avaliação sobre o processo de ensino e de aprendizagem. Dissertação de Mestrado, Universidade Estadual Paulista, Bauru (2016).

[42] J.P. Schardosim, J.P.C. Lima, W. Rochadel e J.B. Silva, RENOTE-Revista Novas Tecnologias na Educação 11, 1 (2013).

[43] I.N. Oliveira, V.D. Chaves, M.G. Prado, C. Takiya e M.S.S. Lopes, Experiências em Ensino de Ciências 11, 3 (2016).

[44] C.R.S. Oliveira, I.N. Oliveira, A.L. Pereira e H.L. Santos, Revista Brasileira de Informática na Educação 17, 1 (2009).

[45] C.A. Santos, Rev. Bras. Ens. Fis. 17, 107 (1995).

[46] M. McRoberts, Arduíno Básico (Novatec Editora Ltda, São Paulo, 2015).

[47] M.A. Cavalcante, C.R.C. Tavolaro e E. Molisani, Rev. Bras. Ens. Fis. 33, 4503 (2011).

[48] A.R. Souza, A.C. Paixão, D.D. Uzêda, M.A. Dias, S. Duarte e H.S. Amorim, Rev. Bras. Ens. Fis. 33, 1702 (2011).

[49] H.P. Cordova, C.E. Aguiar, H.S. Amorim, K.S.O. M. Sathler e A.C.F. Santos, Rev. Bras. Ens. Fis. 40, e2505 (2018).

[50] F.S. Rocha, G.F. Marranghello, M.M. Lucchese, Caderno Brasileiro de Ensino de Física 31, 1 (2014).

[51] J.T. Guaitolini Junior, G.S. Ramos, S.L. Silva e A.C. Gama, Caderno Brasileiro de Ensino de Física 33, 2 (2016).

[52] B. Fry e C. Reas, Getting Started with Processing (Ed. Maker Media, Sebastopol, 2015), $2^{\circ}$ ed.

[53] https://labduino.blogspot.com/search/label/Processing, acessado em 25/02/2018.

[54] BRASIL. Lei $n^{\circ}$ 9.394, 20 de dezembro de 1996. Brasília, 1996. Disponível em: https://www2.camara.leg.br/legin/fed/lei/2017/lei-13415-16-fevereiro-2017-784336publicacaooriginal-152003-pl.html.

[55] BRASIL. Lei $\mathrm{n}^{\circ}$ 13.415, 16 de fevereiro de 2017. Brasília, 2017. Disponível em https://www2.camara.leg.br/legin/fed/lei/2017/lei-13415-16-fevereiro-2017-784336publicacaooriginal-152003-pl.html. 